\title{
DESIGUALDAD, PROPIEDAD PRIVADA Y PENSAMIENTO ECONÓMICO EN EL LARGO PLAZO
}

\author{
Inequality, Private Property and Economic Thought in the Long Term
}

\author{
Adrián Allende Espinosa \\ adrianlles@hotmail.com \\ Universidad de Valladolid. España \\ David Carvajal de la Vega \\ david.carvajal@uva.es \\ Universidad de Valladolid. España
}

Fecha de recepción: 13/09/2017

Fecha de aceptación: 27/09/2017

RESUMEN: El interés actual sobre la desigualdad, acentuado como consecuencia de la crisis económica, no es algo novedoso entre los autores vinculados a la Historia del Pensamiento Económico occidental. El presente estudio realiza un recorrido, desde la Antigüedad a nuestros días, en el que se expone la visión que los principales representantes del pensamiento económico mantenían respecto a la desigualdad. En particular, el trabajo trata de establecer si en este largo camino existe una vinculación clara entre la desigualdad y el triunfo de la propiedad privada en los sistemas económicos occidentales. A través del análisis de textos originales, así como de las consideraciones de los especialistas en el estudio del pensamiento económico, el trabajo muestra una relación, más o menos evidente, entre ambas realidades. Aunque no faltan críticas contra la consolidación de la propiedad privada y la existencia de desigualdad, la primera ha sido plenamente aceptada e integrada en los sistemas económicos contemporáneos, apenas hay debate o cuestionamiento sobre ella. Por otro lado, la desigualdad presenta un panorama diferente. A pesar de reconocer su vigencia y de ser uno de los temas de mayor interés entre la academia, existen diferentes posiciones sobre su origen, su evolución y sobre el nivel de desigualdad de nuestro mundo. El debate en torno a la desigualdad, lejos de agotarse, supone una propuesta atractiva para futuras investigaciones.

Palabras clave: Desigualdad; propiedad privada; pensamiento económico; historia económica.

ABSTRACT: The current interest about inequality, heightened as a consequence of the economic crisis, is not new among authors linked to the History of Western Economic Thought. This study explores 
the views that leading authors of economic thought have held about inequality from Antiquity to our days. In particular, the paper is an attempt to establish a link between inequality and the triumph of private property in Western economic systems in the long term. Through the analysis of original texts and previous works of specialists in the field of economic thought, the study shows a relationship, more or less evident, between both aspects. Although criticism against the consolidation of private property and the existence of inequality abound, the former has been fully accepted and integrated into contemporary economic systems and therefore there is hardly any debate or questioning about it. On the other hand, inequality offers a different outlook. While accepting its presence as a fact, inequality generates great interest among the academy, whose members defend diverse positions regarding its origin, its evolution and today's level of inequality. The debate on inequality, far from being exhausted, is an attractive proposal for future research and reflection.

Keywords: Inequality; Private Property; Economic Thought; Economic History.

SUMARIO: 1. Introducción. 2. Un mundo desigual: el origen y la consolidación de la propiedad privada en la economía occidental, de Aristóteles a la Escolástica. 3. El triunfo de la propiedad privada y el debate sobre el origen de la desigualdad. 4. La expansión de la propiedad privada en un mundo diverso: ¿desarrollo económico o desigualdad? 5. Conclusiones. 6. Bibliografía.

\section{INTRODUCCIÓN}

La desigualdad entre los seres humanos -entendida tanto a nivel social como económico- ha sido objeto de estudio, reflexión y crítica por parte de filósofos, historiadores, economistas y sociólogos, entre otros, a lo largo de la Historia. Pero ha sido durante los últimos años cuando los efectos de la crisis económica han reavivado el debate en torno a la desigualdad y a sus efectos. Esto no supone que la desigualdad haya sido un tema olvidado. Hasta inicios del siglo XXI, los trabajos sobre esta cuestión se centraban mayoritariamente en aspectos metodológicos, como su medición (Atkinson, 1983; Temkin, 1993), o relacionándola con procesos económicos como la globalización (P. H. Lindert, J. G. Williamson, 2003). Hoy, al igual que en el pasado, el interés se centra en analizar sus fundamentos y su evolución a lo largo del tiempo, y en estudiar las consecuencias de este fenómeno en nuestros días. A las diferentes tesis sobre la evolución de la desigualdad planteadas por economistas como B. Milanovic (2012) o T. Piketty (2013) se suman otras obras, como la de J. Stiglitz (2012) que, a un nivel divulgativo, contribuyen al debate y, por qué no, a la polémica en torno al incremento de la riqueza y a la distribución de la misma.

La evolución de la desigualdad durante los últimos dos siglos se ha convertido en uno de los temas de mayor proyección en el mundo académico. No obstante, la desigualdad, y más en particular el estudio de su origen, ha sido clave en el desarrollo de las obras más representativas de la historia del pensamiento económico occidental. Probablemente, uno de los textos más conocidos sea el de J. J. Rousseau, quien, movido por los ideales de la llustración, se atrevió a responder 
a la pregunta planteada por la Academia de Dijon en noviembre de 1753: "Quelle est la source de l'inégalité parmi les hommes, et si elle est autorisée par la loi naturelle?». La interpelación contenía una cuestión de hecho y otra de derecho (Binoche, 2015), esperando que los jóvenes pensadores aclarasen si la Ley Natural sancionaba o no un fenómeno tan propio del hombre como la desigualdad-social, económica y jurídica- desde sus orígenes más remotos. La respuesta de Rousseau, contenida en su Discours sur l'origine et les fondements de l'inégalité parmi les hommes (1754), se centró en explicar la aparición de la desigualdad a partir del surgimiento de una institución esencial en la dinámica económica y social desde entonces: la propiedad privada.

A pesar de la importancia de este tratado, desde cuya aparición han transcurrido dos siglos y medio, la cuestión de la desigualdad y su vinculación con la propiedad no eran asuntos novedosos. Por ello, nos interesa preguntarnos acerca de la visión de otros pensadores, anteriores y posteriores al ginebrino, en torno al origen y evolución de este fenómeno. ¿Podríamos afirmar que la progresiva consolidación de la propiedad privada en las economías occidentales tuvo algo que ver con la evolución de la desigualdad? La respuesta a esta pregunta no es sencilla y la tarea para obtener una respuesta es ingente. Así, el objetivo de este trabajo se basa en realizar un recorrido histórico a través de algunos de los principales representantes de la historia del pensamiento económico, a partir de escritos originales y de otra literatura, vinculando reflexiones en torno a la propiedad privada con el fenómeno de la desigualdad que, en la mayor parte de los casos, quedó en un plano secundario.

Desde Aristóteles hasta la actualidad parece existir una línea argumental coherente, desarrollada por muchos de los pensadores económicos fundamentales en nuestra historia. Aunque en términos generales se ha venido defendiendo la existencia de la propiedad privada con argumentos elaborados y diversos, no es menos cierto que los discursos presentan contradicciones y peculiaridades vinculadas al momento histórico en el que se generaron. Partiendo de la literatura más relevante en lo que a Historia del Pensamiento Económico se refiere, el trabajo se articula en tres grandes bloques relacionados con el origen y la consolidación, el triunfo y la expansión de la propiedad privada. El primero de ellos, la consolidación, se orienta al estudio de los discursos producidos desde la tradición clásica y la escolástica y que, a lo largo de casi dos milenios, asentaron las bases de la propiedad privada en un contexto donde la desigualdad era una realidad. El segundo apartado, el triunfo, hace referencia a las reflexiones planteadas entre los siglos XVII-XIX por parte de humanistas, ilustrados, mercantilistas, clásicos, etc. que marcaron el triunfo definitivo de la propiedad privada en un entorno especialmente complejo, y en ocasiones desigual, que dio lugar a la aparición de la corriente más escéptica respecto a la propiedad privada encabezada por K. Marx. Por último, en el tercer apartado, la expansión, se incluyen algunas de las aportaciones de los pensadores 
y economistas contemporáneos que, tras el triunfo de la propiedad privada en los sistemas económicos occidentales y en la mayor parte de las escuelas de pensamiento económico, se han preocupado por analizar su difusión y consolidación, además de vincular la existencia de propiedad privada con el desarrollo económico y la desigualdad en diferentes países y territorios.

En definitiva, proponemos un recorrido en el largo plazo intentando observar las relaciones entre propiedad privada y desigualdad que, de un modo más o menos claro, han conformado la manera de entender nuestro mundo, nuestra sociedad y nuestro sistema económico.

\section{UN MUNDO DESIGUAL: EL ORIGEN Y LA CONSOLIDACIÓN DE LA PROPIEDAD PRIVADA EN LA ECONOMÍA OCCIDENTAL, DE ARISTÓTELES A LA ESCOLÁSTICA}

Pese a que en la Edad Antigua el pensamiento económico no cobró una relevancia destacada, en esta etapa podemos encontrar planteamientos económicos y sociales que pretendían asentar las bases de diferentes modelos de sociedad. No obstante, como ocurrirá hasta los siglos XVIII-XIX, estos pensadores y los que prosiguen carecían de condiciones suficientes para el estudio de dicho fenómeno socioeconómico.

Los primeros escritos no daban a la desigualdad un papel relevante, puesto que no concebían a esta como un problema -o virtud- que pudiera repercutir de manera drástica en el funcionamiento de sus ciudades-Estado si esta se fundamentaba en el mérito. Mientras Platón sancionaba un modelo de «democracia; y será, según se ve, un régimen placentero, anárquico y vario que concederá indistintamente una especie de igualdad tanto a los que son iguales como a los que no lo son» (Platón, La República, Libro VIII, XI); Aristóteles se expresaba así sobre la igualdad:

Así, la igualdad parece de derecho común, y sin duda lo es, no para todos, sin embargo, sino sólo entre iguales; y lo mismo sucede con la desigualdad; es ciertamente un derecho, pero no respecto de todos, sino de individuos que son desiguales entre sí (...) La desigualdad entre iguales y la disparidad entre pares son hechos contrarios a la naturaleza, y nada de lo que es contra naturaleza puede ser bueno. Pero si hay un mortal que sea superior por su mérito, y cuyas facultades omnipotentes le impulsen sin cesar en busca del bien, éste es el que debe tomarse por guía, y al que es justo obedecer. (Aristóteles, La PolHtica, Libro III, Capítulo V).

Ambos autores concebían y admitían un tipo de desigualdad: la desigualdad natural. Ambos la definen como aquella que viene dada de forma meramente 
natural, es decir, la que se considera como inherente a la condición humana: cada persona tiene unos rasgos, cualidades, virtudes o defectos distintos, por lo que no existe la igualdad plena entre dos individuos. Partiendo de este concepto, en La República, Platón planteaba un modelo de sociedad en el que existían fenómenos de desigualdad, como por ejemplo en la división del trabajo entre los diferentes eslabones de la población -ya planteada anteriormente por Sócrates-. De esta forma, se generaba un reparto de responsabilidades en el que el grupo dominante obtenía los encargos más relevantes, de entre los cuales podemos destacar el de la enseñanza, tratando estos de transmitir a los eslabones inferiores de la sociedad los aspectos nocivos de un hipotético desarrollo de la desigualdad dentro de la polis (Rothbard, 1999, pp. 40-41). Platón formulaba un modelo social desigualmente distribuido como consecuencia de las diferentes cualidades de cada ser humano. No obstante, consciente de los problemas que la desigualdad, sobre todo la económica, podría acarrear, el autor condenaba explícitamente la desigualdad generada por el beneficio de cualquier actividad, como por ejemplo el comercio. Platón planteaba la posibilidad de restringir actividades de intercambio o que pudiesen dar lugar a la generación de cualquier tipo de lucro, intentando asegurar la igualdad total dentro de cada eslabón.

En lo que respecta a la propiedad, los pensadores antiguos utilizaron este concepto para caracterizar sus respectivos modelos de sociedad. Cuestiones como la concesión o la posesión de propiedad privada comenzaron a incorporarse al debate sobre el modelo político, social y económico que debía regir en la antigua Grecia. En este contexto, la relación entre desigualdad y propiedad privada se establecía dependiendo de si esta última estaba o no restringida, lo que implicaba que los Estados contasen con grados de desigualdad más o menos altos. Muchas de estas ideas son extensibles a gran parte de los pensadores que estudiaremos en el presente trabajo, pero, como veremos, a partir de las reflexiones de los pensadores de la Antigüedad se puede considerar que la relación entre la permisividad (o fomento) de la propiedad privada por parte de las élites estatales y la desigualdad era notable.

Los escritos de Platón y Aristóteles ya nos permiten vincular propiedad privada y desigualdad. Como es bien conocido, cada uno planteaba una forma diferente de sociedad basándose en la manera de repartir responsabilidades -o medios- entre cada uno de los «agentes» que forman parte de ella. Sus modelos de sociedad presentaban características sensiblemente diferentes, con mayor o menor grado de desigualdad, dependiendo de la forma en que se entendiese la propiedad privada: como algo a restringir o, por el contrario, como algo natural y permitido. Es importante resaltar que los modelos de sociedad planteados por los filósofos de la Antigua Grecia destacaban por su simplicidad, llevando ello al escaso matiz de términos económicos, basados en su mayoría en la explicación de las necesidades «naturales», entre las que perfectamente encajaría la propiedad. 
Sensiblemente diferente a la visión de Platón, el pensamiento de Aristóteles destacaba por su firme defensa de la propiedad privada ya que, según él, esta permitía una mejor adaptación de su modelo de sociedad a la naturaleza humana. En su Política, Aristóteles realizó un planteamiento que, tras su redescubrimiento en la Plena Edad Media, se convirtió en uno de los argumentos más sólidos utilizados por los defensores de la propiedad privada hasta nuestros días. El pensador griego argumentaba que la propiedad privada era una de las mejores formas de tenencia de la propiedad y, en su defensa, explicaba que el propio interés que cada individuo pone en las cosas que posee conlleva un mejor cuidado de ellas: «lo que es común a un número muy grande de personas obtiene un mínimo cuidado. Pues todos se preocupan especialmente de las cosas propias, y menos de las comunes, o solo en la medida que atañe a uno" (Aristóteles, La Política, Libro II, Capítulo III). Pese a aceptar la desigualdad en su modelo de sociedad, Aristóteles reconocía que no era deseable, ya que surgía como resultado de la acumulación, fenómeno que despreciaba. Con el ánimo de evitar esta situación, el pensador sugería la conveniencia de contar con un modelo educativo que inculcase su rechazo proponiendo, en último término, la eliminación de la acumulación y, por tanto, de la desigualdad (Rothbard, 1999, pp. 43-44).

Tras las reflexiones planteadas por Platón y Aristóteles apenas existen aportaciones que muestren nuevos enfoques sobre la propiedad privada y la desigualdad hasta avanzada la Edad Media. Autores como J. Schumpeter (2015) o M. Rothbard (1999) coinciden en la existencia de más de un milenio de silencio y de ausencia de grandes aportaciones al pensamiento económico y, menos aún, en lo que se refiere a las dos cuestiones centrales en este trabajo. Hasta la aparición de la Escolástica en la Edad Media es difícil reseñar grandes aportaciones al pensamiento económico. Aunque muchas de las ideas fruto de esta escuela fundada sobre la doctrina cristiana se centraban en el modelo social y político, los autores representantes de la escolástica también expusieron en sus obras cuestiones relativas a la propiedad privada y la desigualdad.

Desde el siglo XIII, Tomás de Aquino, principal referente de esta corriente que redescubría la tradición aristotélica, introdujo en sus escritos reflexiones económicas sobre la propiedad privada legitimando la existencia de la misma. La mayor diferencia de sus planteamientos con los del pasado se centraba en su explicación del origen de la propiedad. A pesar de defender sus bondades, Santo Tomás -y otros escolásticos- no llevaron a cabo una defensa férrea de esta institución; aunque sí aclaraban que el origen de la misma se encontraba en Dios, de quien emanaba la sociedad y el sistema económico en el que se reconocía la propiedad privada. Dios era el poseedor de los bienes exteriores y los seres humanos, hechos a su imagen y semejanza, podían disponer de ellos y disfrutarlos en su privacidad. Así respondía Tomás de Aquino a la cuestión sobre el origen de la propiedad, dejando en manos de los hombres el dominio natural de las cosas: 
¿Cuál es el origen de la propiedad privada?

«Las cosas exteriores pueden considerarse de dos maneras: una, en cuanto a su naturaleza, la cual no está sometida a la potestad humana, sino solamente a la divina, a la que obedecen todos los seres; otra, en cuanto al uso de dichas cosas, y en este sentido tiene el hombre el dominio natural de las cosas exteriores, ya que, como hechas para él, puede usar de ellas mediante su razón y voluntad en propia utilidad». (T. de Aquino, Sum. Theo. II, 2, cuestión LXVI, art. 1).

El hecho de que los bienes puedan ser considerados como propiedad privada traería consigo los beneficios expuestos anteriormente por Aristóteles: mayor cuidado y esmero en su explotación y la garantía de contar con un orden social donde debía reinar la paz que, a su vez, se convertía en uno de los objetivos de la teoría social. Así lo expresa Tomás de Aquino, quien considera lícita la posesión por tres motivos: por una mejor gestión de los bienes, por una administración más ordenada derivada del propio interés y por mantener un estado de paz entre los hombres.

¿Es lícito poseer una cosa como propia?

«Acerca de los bienes exteriores, dos cosas le competen al hombre. La primera es la potestad de gestión y disposición de los mismos, y en cuanto a esto, es lícito que el hombre posea cosas propias. Y es también necesario a la vida humana por tres motivos: primero, porque cada uno es más solícito en gestionar aquello que con exclusividad le pertenece que lo que es común a todos o a mucho [...]; segundo, porque se administran más ordenadamente las cosas humanas si a cada uno le incumbe el cuidado de sus propios intereses; sin embargo, reinaría confusión si cada cual se cuidara de todo indistintamente; tercero, porque así el estado de paz entre los hombres se mantiene si cada uno está contento con lo suyo». (T. de Aquino, Sum. Theo. II, 2, cuestión LXVI, art. 2).

Tomás de Aquino reconocía que la propiedad es un concepto creado por el ser humano y que esta no es contraria al Derecho Natural. Justifica nuevamente, desde un enfoque individualista, que el propietario de un bien deposita mayor cuidado sobre él, suponiendo asimismo un incentivo y evitando posibles disputas. Además, su defensa de lo privado se basa en la garantía de un orden social derivado de la gestión particular de los recursos propios, de forma que no existieran conflictos derivados de la puesta en común de todas las cosas. Por lo tanto, podemos concluir que su defensa de la propiedad privada tiene como fin alcanzar la estabilidad social; cuestión controvertida, ya que exigiría que todos y cada uno de los hombres alcanzasen un nivel de propiedad aceptable.

Casi a la par que el teólogo dominico expresaba sus ideas en torno a la propiedad, otras corrientes dentro del cristianismo lo hacían de un modo más crítico. En particular, un grupo de Franciscanos, encabezados por Francisco de Soto, se mostraron contrarios 
a la propiedad privada -entendida como una creación exclusiva de los hombres-y, en particular, rechazaron la acumulación de bienes por parte de unos pocos. Cabe decir que las posturas críticas fueron condenadas y rectificadas a través de textos como la bula Quía vir reprobus (1329, Juan XXIII), donde se aceptaba la propiedad privada y, de nuevo, vinculaban su origen a Dios y no a la convicción humana.

Ya en el siglo XVI, la Escuela de Salamanca propuso una defensa férrea de la propiedad privada en base a la tradición escolástica. Algunos de sus representantes como L. de Molina, F. de Vitoria o D. de Soto se encuentran entre los primeros teóricos del pensamiento económico que se posicionaron de forma clara ante esta cuestión (San Emeterio, 2002). En general, los autores vinculaban la existencia de propiedad privada a la Ley Natural, situando su origen en un proceso inicial de apropiación y división de los bienes. Esta visión apenas cambiaría en el futuro, al menos en lo fundamental, aunque una vez delimitado el derecho de gentes, situado entre el Derecho Natural y el Derecho Civil, pensadores como F. Vitoria y D. de Soto pudieron perfilar sus teorías sobre el origen y justificar con mayor solidez la existencia de propiedad privada. Esta cuestión es sumamente importante, ya que la mayor parte de los teóricos sitúan el origen de la propiedad privada en un estadio anterior al del nacimiento del Derecho Civil y del gobierno, otorgando a esta institución una importancia superior a otras al surgir en la primera etapa del desarrollo de la sociedad civil.

La defensa que hacía F. de Vitoria de la propiedad privada se fundamentaba, al igual que ocurría en el caso de Aristóteles y de Tomás de Aquino, en un mejor cuidado de los bienes cuando estos se encuentran en manos privadas, afirmando "que la comunidad de bienes requiere muchas otras cosas que no suelen hallarse en la sociedad. Requiere modestia, la concordia, la debida sujeción y la justa distribución, las cuales no existirían si todas las cosas fuesen comunes» (Vitoria, F., 1937: Cuestión 66, a.2, vol. 2) (San Emeterio, 2002, p. 36). Las dudas del autor sobre la viabilidad de la comunidad de bienes son compartidas por D. de Soto, quien, en una línea similar a la de Vitoria, afirmaba que «si hubiera comunidad de bienes desaparecería la virtud de la liberalidad; lo cual no es pequeño esplendor para la nación (...) Y de esta manera desaparecería la virtud de la hospitalidad, ni se atendería a los peregrinos, ni se socorrería a los necesitados; y como consecuencia desaparecería la virtud del agradecimiento por los beneficios recibidos» (Soto, D., 1968: Libro IV, q.3, a.1). En definitiva, la propiedad privada, siempre disponible para el uso comunitario en caso de necesidad y de urgencia, era la mejor manera de preservar el bien común y la concordia sin llegar a ser cuestionada por fenómenos como la desigualdad, ya visible durante este período.

A modo de resumen, podemos sostener que, desde los primeros textos antiguos hasta los escritos de los escolásticos, la defensa de la propiedad privada se basó en justificarla como uno de los mejores medios para lograr un orden social estable, garantizando además el cuidado óptimo de cada bien, en contraposición con aquellos que estaban dispuestos en común. 


\section{EL TRIUNFO DE LA PROPIEDAD PRIVADA Y EL DEBATE SOBRE EL ORIGEN DE LA DESIGUALDAD}

Aunque la Escolástica supuso un impulso importante a los planteamientos teóricos favorables a la propiedad privada, sustentándola sobre justificaciones teológicas relacionadas con el bien común, sobre el Derecho Natural o sobre el derecho de gentes, la propiedad privada continuó siendo objeto del interés y polémica entre muchos pensadores que, durante los siglos XVII y XVIII, difundieron sus ideas al calor de las nuevas corrientes humanistas e ilustradas. Autores del entorno inglés y holandés como Th. Hobbes, J. Locke o H. Grocio, especialmente cercanos al Derecho Natural, además de los franceses y escoceses, de los que hablaremos más adelante, son ejemplo del interés por este tema y, cómo no, por la desigualdad.

En lo que respecta a los humanistas y a la desigualdad, muchos hacen mención a una etapa de igualdad entre los seres humanos, al menos en lo que se refiere a un estadio inicial de nuestra historia. La desigualdad vendría después, y estaría generada, según los defensores del igualitarismo analítico, como Hobbes, por el desigual desarrollo que cada ser humano hace de unas capacidades que, en principio, eran similares. Como vemos, ideas que nos recuerdan a los planteamientos de los pensadores de la antigua Grecia. A diferencia de este enfoque, los defensores del igualitarismo normativo vinculaban la desigualdad al incumplimiento del precepto, según el cual, todos los seres humanos son iguales por ser hijos de Dios, creados a su imagen y semejanza.

Como contrapunto a Hobbes y a su Leviathan (1651), J. Locke se preocupó por ensalzar al individuo y por explicar cómo surgió la desigualdad entre los hombres que habían partido de un estadio de igualdad inicial. Sus reflexiones, principalmente las contenidas en sus Dos tratados sobre el Gobierno Civil (1690), pasan por reconocer y defender el derecho de la propiedad privada que, en último grado, iba a generar desigualdad económica y social entre los hombres. En su razonamiento, la propiedad no derivaba de un acuerdo social que permitía el tránsito entre el estadio natural y una sociedad organizada, sino que dependía del sujeto (Roncaglia, 2006, pp. 120121), mostrando claramente una visión más individualista que la de Hobbes y su modelo. Basándose en el desarrollo que cada ser humano hace de sus capacidades y teniendo en cuenta que todo hombre nace con «un derecho a la libertad de su persona, sobre la cual ningún otro hombre tiene poder, y de la que puede disponer libremente ${ }^{1}$, Locke argumentaba que las personas son propietarias de su trabajo y, por ende, de los resultados del mismo. Bajo dos axiomas, el de la "autoposesión» -uno es dueño de su trabajo- y el de la propiedad derivada del "primer uso" u "ocupación", Locke defendía que una persona tenía derecho a obtener la posesión privada de un bien al haber invertido su trabajo en la explotación del mismo ${ }^{2}$. En

\footnotetext{
1 J. Locke, Segundo tratado de gobierno, Cap. 16, «De la conquista» - n. 190.

2 Ibid., Cap. 5, «De la propiedad» - sobre la propiedad y el trabajo.
} 
definitiva, las pesquisas y reflexiones en torno a la desigualdad seguían centrándose en la naturaleza de los hombres y en la capacidad de apropiación de estos en algún momento de la historia (Schumpeter, 2015).

La complejidad de la reflexión teórica sobre la propiedad privada y la desigualdad -como vemos, estrechamente relacionada con el orden social- hizo necesarias nuevas aportaciones con un carácter más amplio y a la vez integrador, centradas en el hombre y en su historia, a la vez que Dios era relegado del primer plano. Es en este contexto, ya en el siglo XVIII, cuando destacan las aportaciones de los fisiócratas e ilustrados franceses y de los «economistas» escoceses.

Los fisiócratas franceses, como F. Quesnay o P. P. Mercier de la Rivière, mantuvieron planteamientos próximos a los que acabamos de ver, si bien eran especialmente cercanos a la tradición iusnaturalista. En términos generales, estos autores reconocían el derecho individual de las personas, derivado de las Leyes Naturales y, por tanto, se mostraban proclives al derecho a la propiedad privada al que consideraban parte del «orden natural». En su pensamiento existe una clara vinculación entre propiedad y libertad, otorgando al gobierno la función de defender ambas a través del reconocimiento y sanción del derecho de libertad individual que implicaba la propiedad. Las dos condiciones se antojaban necesarias para el desarrollo de otras actividades como el intercambio comercial (Rothbard, 1999, pp. 410-411), logrando así el progreso de la economía y de la sociedad.

Otros autores, también vinculados al Derecho Natural, realizaron planteamientos no del todo coincidentes con los que acabamos de citar. N. de Condorcet, por ejemplo, expuso su defensa de la idea de progreso ligado a la igualdad, una idea que pocos habían valorado; más bien al contrario, habían justificado la desigualdad generada por las diversas vías de acceso a la propiedad privada que, en última instancia, sería beneficiosa para el bien común. Bajo estas premisas e ideas surgen los autores que Schumpeter define como "semisocialistas" (2015, pp. 179-180). Entre los autores citados en este grupo merece la pena destacar a G. Bonnot de Mably, pensador que daba respuesta directa a la pregunta que venimos desarrollando en este trabajo, al plantear que la desigualdad era consecuencia de la existencia de propiedad privada. Menos explícitos fueron los planteamientos de otros como J. J. Rousseau o J. P. Brissot de Warville. Este último se mostró especialmente crítico con la propiedad privada esgrimiendo que no existe un derecho a la propiedad privada, pues la consideraba un robo. Menos «radicales» eran los planteamientos de Rousseau. En su Discurso sobre el origen de la desigualdad entre los hombres, el autor se preocupó por mostrar el origen de la desigualdad a través del análisis del "hombre salvaje» en los estadios iniciales de la historia, en el momento de aparición de la propiedad y de la posterior organización de la sociedad civil. Es conveniente destacar que para Rousseau los primeros procesos de apropiación estaban ligados a la aparición de la sociedad civil: «el primer hombre a quien, cercando un terreno, se lo ocurrió decir esto es mío y halló gentes bastante simples para creerle fue el verdadero fundador de la sociedad 
civil» y que solo cuando la acumulación de bienes se mostró útil para los hombres, estos promovieron la desaparición de la igualdad originaria:

desde el instante en que mi hombre tuvo necesidad de la ayuda de otro; desde que se advirtió que era útil a uno solo poseer provisiones por dos, la igualdad desapareció, se introdujo la propiedad, el trabajo fue necesario y los bosques inmensos se trocaron en rientes campiñas que fue necesario regar con el sudor de los hombres y en las cuales viose bien pronto germinar y crecer con las cosechas la esclavitud y la miseria ${ }^{3}$.

A pesar del escepticismo expresado por estos autores, el reconocimiento de la propiedad privada como elemento clave en el sistema económico occidental era una realidad y así lo acabaron sancionando las obras de autores como D. Hume o A. Smith, conocedores del pensamiento ilustrado y, en particular, de los autores franceses. Hume, basándose en sus predecesores, realiza interesantes aportes a la teoría de la propiedad, renegando de los postulados del Derecho Natural del que también era heredero (San Emeterio, 2002, pp. 725 y ss.).

El pensador escocés, interesado en diseccionar la naturaleza humana, fundamentaba su explicación sobre el origen y la evolución de la propiedad en elementos que a su juicio caracterizan al ser humano. Por lo tanto, no pone tanto interés en el origen y en la evolución temporal de la propiedad y de las desigualdades generadas en torno a ella; Hume se centra en explicar los elementos que han generado e impulsado la propiedad privada: la escasez y la generosidad limitada que, a su vez, están íntimamente ligados a la desigualdad económica. A partir de este punto, el proceso de apropiación de bienes se convirtió en un proceso complejo que no responde a la lógica expresada por muchos autores, como el propio Locke, que lo vinculaban a la posesión con el trabajo y la inversión de recursos. Hume consideraba que la apropiación podía llevarse a cabo por mérito, pero también de forma arbitraria. Tan solo en el largo plazo parecía detectar una tendencia a la concentración -reconociendo de este modo la existencia de desigualdad-, que se realizaría entre los más meritorios a través de la transmisión y gracias a la defensa efectiva por parte de la ley y la justicia, velando siempre por el interés de la sociedad (Venning, 1976, pp. 80-81): «Nuestra propiedad no es más que los bienes cuya constante posesión está establecida por las leyes de la sociedad, o sea por las leyes de la justicia» (Hume, Tratado de la naturaleza humana, Parte Segunda, De la justicia y propiedad, Sección II: Del origen de la Justicia y la Propiedad).

${ }^{3}$ Rousseau, J. J. (1754). Discurso sobre el origen de la desigualdad entre los hombres. http://www.cervantesvirtual.com/obra-visor/discurso-sobre-el-origen-de-la-desigualdadentre-los-hombres--0/html/ff008a4c-82b1-11df-acc7-002185ce6064_9.htm (fecha de consulta: 27/07/2017). 
Muy cercano a los planteamientos de Hume, aunque no en todo, A. Smith también dedicó parte de sus pesquisas y reflexiones a la cuestión de la propiedad privada con el fin de entender su relación con la práctica del derecho, así como su aporte a los sistemas económicos occidentales y, en último término, a la riqueza de las naciones y al desarrollo del liberalismo económico. La postura de Smith respecto a la propiedad privada, como ocurre con otros autores, parece desligarse del Derecho Natural, aunque no de modo claro. En sus Lecciones de Jurisprudencia, Smith identificaba el derecho a la propiedad como una de las leyes fundamentales de la justicia dentro de la sociedad (San Emeterio, 2002, p. 304), es más, la existencia de la propiedad civil y la necesidad de defenderla a través de la justicia -similar a lo planteado por Hume- se encontraban en el origen del gobierno civil.

Smith era consciente de la relación entre propiedad privada y desigualdad que vinculaba a la existencia de ricos y pobres-. El ejemplo que cita el autor parte de un estado de igualdad entre los miembros de una nación de cazadores donde no existía la propiedad privada ni tampoco gobierno, pues no era necesario. A partir del proceso de apropiación de bienes en el origen, Smith proponía una explicación sobre cómo se desarrolló la propiedad privada. Así, procede a diferenciar a aquellos que poseen -ricos- de los que no poseen y desean poseer -pobres-, aunque las diferencias no quedan aquí. La «desigual fortuna» de ricos y pobres será el germen de la dependencia de los segundos respecto a los primeros, profundizando así en la desigualdad. Esta situación, junto con el deseo de los pobres por poseer, ejercerán como desencadenantes de la violencia que pondrá en peligro la estabilidad en la sociedad, objetivo último que los pensadores ansiaban desde la Antigua Grecia. Ante esta situación, Smith consideraba que la aparición del gobierno y de instituciones como la justicia eran necesarias. Es más, entre los fines de ambas instituciones se encontraba la protección de la propiedad privada y, por ende, asegurar la estabilidad del sistema.

The appropriation of herds and flocks which introduced an inequality of fortune, was that which first gave rise to regular government. Till there be property there can be no government, the very end of which is to secure wealth, and to defend the rich from the poor. In this age of shepherds, if one man possessed 500 oxen, and another had none at all, unless there were some governments to secure them to him, he would not be allowed to possess them. This inequality of fortune, making a distinction between the rich and the poor, gave the former much influence over the latter, for they who had no flocks or herds must have depended on those who had them, because they could not now gain a subsistence from hunting, as the rich had made the game, now become tame, their own property. (Adam Smith, Lectures on Justice, Police, Revenue and Arms, delivered in the University of Glasgow. Reported by a Student in 1763). 
El autor escocés parecía tener claro que propiedad privada y desigualdad habían avanzado de la mano a lo largo de la historia, si bien nunca se mostró contrario a la existencia de la misma, ya que consideraba a la primera un elemento potenciador en el desarrollo de las economías que tuvo ocasión de estudiar.

Entre las ideas desarrolladas por Smith destaca su explicación sobre el nacimiento de la propiedad a través del paso de la era de los cazadores a la era de los pastores, etapa que supuso la aparición de diversas formas de apropiación y de los gobiernos, cambiando radicalmente una situación que se prolongó a lo largo de las siguientes eras, de los agricultores y del comercio. Fue en estas etapas cuando se consolidaron otras formas de apropiación como la tradición, la accesión, la prescripción o la sucesión. Otro de los planteamientos más interesantes de Smith es el que se refiere al carácter flexible o "cambiante» de la propiedad privada. Según el pensador, esta no tenía por qué mantenerse siempre en las mismas manos y, además, podía mutar. De este modo, en el largo plazo, la propiedad tendería a concentrarse en manos de los más capaces y, bajo ningún concepto, debía imponerse el derecho a la propiedad ante las necesidades extremas de una sociedad, como ocurría con las hambrunas: ante el hambre, en cierto modo era razonable asaltar un granero. Como ejemplo de esta flexibilidad que caracterizaba a la propiedad privada, Smith se mostraba contrario a las instituciones que impedían la enajenación de bienes, como ocurría en el caso de los entails o de los mayorazgos, o que primaban la transmisión de la misma en base a criterios que poco o nada tenían que ver con la capacidad y el mérito como, por ejemplo, el hecho de heredar por ser primogénito.

En definitiva, a finales del siglo XVIII, con la ciencia económica en proceso de consolidación, apenas nadie discutía el derecho a la propiedad privada. Las virtudes de su existencia: asegurar el bien común, preservar los bienes a través de su cuidado, incentivar el desarrollo económico a través de la posesión, asegurar una buena gestión y administración de los recursos, etc. suponían mayores beneficios que los prejuicios derivados de la desigualdad entre las personas. No obstante, aunque eran pocos los que se atrevían a criticar o, al menos, a discutir el derecho a la propiedad privada, su influencia fue notable, como ocurrió en el caso de K. Marx.

Los autores más críticos y, en alguna medida, antecesores de los planteamientos del pensador alemán se retrotraen a la existencia de corrientes comunistas primitivas, en ocasiones con tintes milenaristas (Rothbard, 1999, pp. 329-340). Desde el siglo XVI, autores como T. Moro y su Utopía (1516) o, ya en el siglo XVII, G. Winstanley, en su The New Law of Righteousness (1649) proferían críticas contra la desigualdad, contra la propiedad privada y el egoísmo imperante entre los hombres. Este tipo de visiones que clamaban contra la desigualdad calaron en otros autores como G. Bonnot de Mably, según el cual era imperativo regresar al estado natural en el que todos los hombres son iguales -regresar al comunismo primitivo-, prescindiendo de la propiedad privada que, vista como una anomalía, permitía a unos pocos 
indolentes vivir del resto de la sociedad. Esta corriente de pensamiento enraizó en algunos autores que, ante la imposibilidad de acabar con una institución como la propiedad privada, generadora de desigualdad, pero integrada en el hombre moderno, promovían un cambio a modo de "revolución», como procuró F. Babeuf en 1796. A través de la Conjuration des Égaux, el francés intentó promover la igualdad absoluta imponiendo, entre otras medidas, la comunidad de la propiedad que debía acabar con la injusticia generada por la desigualdad.

Tras estos autores a los que poca atención se ha dedicado, K. Marx es sin duda uno de los pensadores más influyentes en lo que a la desigualdad económica y social se refiere. Valiéndose de planteamientos anteriores como los de Locke, al defender que cada uno es propietario de su trabajo y que a través de este se puede acceder a la propiedad privada, el pensador alemán entendía que un proletario también debía ser propietario de su trabajo y así podría acceder a la propiedad. Así lo expresaba Marx (1932) en sus Manuscritos económico-filosóficos de 1844, antecedentes de su pensamiento ${ }^{4}$. No obstante, el problema que planteaba Marx era complejo y en cierto modo respondía a fenómenos reales, ya que el sistema había privado a los proletarios de la posesión de su trabajo y, por tanto, les impedía acceder a la propiedad y convivir en condiciones de igualdad.

El siguiente paso de Marx y de sus seguidores tuvo su reflejo en el Manifiesto Comunista (1848). En él, sus partícipes se encargaron de aclarar que el comunismo no pretendía acabar con propiedad preexistente. Ahora bien, la lucha contra la propiedad privada se había convertido en piedra de toque de este movimiento, ya que esta forma de propiedad era representativa del último estadio en la evolución de los regímenes de propiedad, encumbrado a la propiedad burguesa como la más elaborada expresión de la desigualdad entre los hombres.

La supresión del régimen de propiedad preexistente no es la característica específica del comunismo. Todos los sistemas de propiedad históricos estuvieron sujetos a continuos cambios, a la continua modificación de la historia. La Revolución francesa, por ejemplo, abolió la propiedad feudal en favor de la propiedad burguesa. Lo que caracteriza específicamente al comunismo no es la supresión de la propiedad en general, sino la supresión de la propiedad burguesa (...) la expresión última y más acabada de la producción y apropiación de productos basadas en los antagonismos de clase, en la explotación de unos hombres por otros. En este sentido, los comunistas sí que podrían resumir sus teorías en esta sola fórmula: supresión de la propiedad privada. (K. Marx y F. Engels, 1848, Manifiesto Comunista, II. Proletarios y comunistas).

4 De los Cuadernos de París, así conocida esta obra, hay que destacar el Tercer Manuscrito y, en concreto, el apartado dedicado a «Propiedad privada y trabajo. Economía política como producto del movimiento de la propiedad privada». 
El problema pues no se encontraba como tal en el concepto de propiedad, sino en la propiedad burguesa y en la idea de capital como representación de la apropiación del trabajo de los proletarios. La pretensión de Marx y de Engels, al menos en su origen, se orientaba a colectivizar el capital, lo que «no significa que la propiedad personal se transforme en colectiva; solo queda transformado el carácter social de la propiedad al perder el carácter de clase» (Marx y Engels, 1848).

Ante la defensa de la propiedad privada como precepto generalizado en el modelo social y económico imperante, Marx criticaba la contradicción del sistema ya que gran parte de la sociedad no tenía posibilidades de acceder a la propiedad privada. El sistema, y en particular los más ricos, lo impedían a través de la expropiación del trabajo y de la propiedad privada de gran parte de la sociedad. Por ello, no consideraba traumático suprimir este sistema que, en lo esencial, fomentaba la desigualdad entre las personas.

Os aterráis de que queramos suprimir la propiedad privada como si no fuese una realidad que en la sociedad actual, la vuestra, se ha suprimido la propiedad privada para el noventa por ciento de sus miembros. La propiedad que existe se basa precisamente en su no existencia para ese noventa por ciento. Lo que nos reprocháis, pues, es querer suprimir una propiedad que tiene como condición necesaria la carencia de propiedad de la aplastante mayoría de la sociedad. Nos reprocháis, en una palabra, el querer suprimir vuestra propiedad. Ciertamente, es eso lo que pretendemos. (K. Marx y F. Engels, 1848, Manifiesto Comunista, II. Proletarios y comunistas).

En definitiva, el discurso de Marx y de otros pensadores de su entorno suponía una de las críticas más duras contra la propiedad privada a mediados del siglo $\mathrm{XIX}$, pues esta se había convertido en propiedad burguesa y había generado una profunda situación de desigualdad. Ante esta propiedad, concentrada en pocas manos y caracterizada por arrebatar al proletariado la propiedad de su trabajo, no cabía una defensa bajo los tradicionales argumentos del bien común o de la paz social; ya no eran suficientes para defender el sistema de propiedad privada tal y como se había desarrollado hasta ese momento. No obstante, y a pesar de la importancia que este movimiento tuvo y ha tenido a lo largo del último siglo y medio, el triunfo de la propiedad privada como institución fundamental de los sistemas económicos occidentales no tenía vuelta atrás.

La visión que nos aportan diferentes pensadores a través del estudio y la comprensión de los fenómenos que aquí nos interesan nos permite reconocer un claro avance en cuanto a la conceptualización de la propiedad privada y de la desigualdad, si bien no siempre es fácil establecer siempre una relación directa entre propiedad privada, concentración de la riqueza, desarrollo económico y generación de desigualdad. Hasta la fecha, los estudios empíricos que han tratado el mundo preindustrial muestran la existencia de desigualdad (van Zanden, et al., 
2012; Alfani, 2015; Reis, 2017), si bien su evolución hasta nuestros días ha sido y continúa siendo objeto de controversia, como veremos más adelante. Algunos autores se han atrevido incluso a comparar diferentes economías en su estadio pre-industrial, concluyendo que la desigualdad se asemeja bastante en esta etapa sin importar el momento histórico en el que se haya dado (Milanovic, et al., 2011). En cualquier caso, la desigualdad en las economías occidentales entre los siglos XVII-XIX era una realidad que, para la mayor parte de los pensadores, perduró en el tiempo.

\section{LA EXPANSIÓN DE LA PROPIEDAD PRIVADA EN UN MUNDO DIVERSO: ¿DESARROLLO ECONÓMICO O DESIGUALDAD?}

La expansión del modelo económico occidental a lo largo y ancho del globo trajo consigo la inevitable expansión de la propiedad privada. Durante el siglo XIX, el proceso de expansión internacional de las economías occidentales -sobre todo de las europeas- a través de fenómenos como el colonialismo o la globalización favoreció el reconocimiento -con mayor o menor éxito- de esta institución en otros territorios donde, al parecer, esta cuestión no había llegado a generar debates como los que hemos venido reproduciendo en páginas anteriores.

En las metrópolis europeas, aunque la mayor parte de los teóricos habían asumido la existencia y la necesidad de esta institución, no faltan testimonios como los de J. S. Mill interesados en incluir sus reflexiones sobre la necesidad de mejora de esta institución (Perky, 2016, pp. 69 y ss). Así se aprecia en escritos como los Principles of Political Economy (1848), tildados por muchos de socialistas, en los que defiende un cambio en la propiedad privada que, hasta entonces, se había desarrollado de un modo imperfecto (Légé, 2006). El utilitarismo, rasgo que define buena parte de la obra de Mill, debería guiar el desarrollo de la propiedad privada.

Desde los escritos del siglo XIX, poco más relevante hemos podido rescatar sobre la propiedad privada y la desigualdad entre las grandes aportaciones a la historia del pensamiento económico. Esto no quiere decir que durante este período la propiedad privada fuese una institución incuestionable, como se aprecia en los procesos de nacionalización vividos a raíz de la I Guerra Mundial o a través de la irrupción del Comunismo en Rusia tras los acontecimientos de 1917.

Más allá de los movimientos políticos, los teóricos mantuvieron las posturas heredadas del siglo XIX, ya fuesen a favor o en contra de la propiedad privada. Como ejemplo, podemos citar la defensa a ultranza de los economistas austriacos. H. Gossen o L. von Mises, por ejemplo, desarrollaron su discurso sobre las bases de un modelo de sociedad y de economía que solo podría evolucionar de forma adecuada bajo una defensa clara de la propiedad privada (Holcombe, 2014, pp. 
105 y ss.). Es más, Mises consideraba que solo bajo un sistema que asegurase la propiedad privada perfecta los agentes podrían contar con plena capacidad de actuación y, en consecuencia, actuar y tomar decisiones en las mejores condiciones posibles. Estos discursos pretendían luchar contra los procesos de nacionalización y, a la vez, contraponer las ideas defendidas desde el Comunismo y sus teorías sobre la apropiación y la redistribución de la riqueza. Como vemos, la desigualdad, o al menos el análisis empírico de la misma, quedaba en un segundo plano frente a la actividad política. Excepciones como las del propio Mises (On Equality and Inequality, 1961) apenas profundizan en una cuestión que no se antojaba excesivamente problemática.

En lo que respecta a la desigualdad, hay que reconocer que diversos acontecimientos del siglo XX-XXI han reavivado el interés por ella. En particular, las crisis económicas y la generación de pobreza motivaron escritos como el de J. M. Keynes al concluir su Teoría General (1936). En su libro, Keynes reconocía la existencia de desigualdad y, en cierto modo, la argumentaba bajo razonamientos similares a los vistos en el anterior apartado (capacidades diferentes, acumulación, etc.), aceptando un modelo en el que la propiedad privada ejercía como estímulo. Pero Keynes ponía el acento en las desigualdades excesivas de su tiempo que, sin justificación, tiranizaban a los hombres, provocando que unos pocos sometiesen al resto.

Los principales inconvenientes de la sociedad económica en que vivimos son su incapacidad para procurar la ocupación plena y su arbitraria y desigual distribución de la riqueza y los ingresos (...) Por mi parte creo que hay justificación social y psicológica de grandes desigualdades en los ingresos y en la riqueza, pero no tan grandes disparidades como existen en la actualidad. Hay valiosas actividades humanas cuyo desarrollo exige la existencia del estímulo de hacer dinero y la atmósfera de la propiedad privada de la riqueza (...) Es preferible que un hombre tiranice su saldo en el banco que a sus conciudadanos; y aunque se dice algunas veces que lo primero conduce a lo segundo, en ocasiones, por lo menos, es una alternativa. (J. M. Keynes, Teonнa General de la ocupaciyn, el interŭs y el dinero, 1934, cap. 24).

Avanzado el siglo $\mathrm{XX}$, a medida que el institucionalismo económico cobraba importancia, los estudiosos recuperaron el interés por destacar las bondades de la propiedad privada (North, 1990). Los análisis derivados de esta corriente, sobre todo los centrados en el crecimiento y el desarrollo económico (Acemoglu, et al., 2005) han puesto énfasis en las diferencias entre países ricos y pobres, teorizando sobre el origen de las mismas. Buena parte de estos trabajos, vigentes en nuestros días, han consolidado el marco de análisis previo, sancionando la relación entre propiedad privada -su efectiva aplicación y defensa- y el desarrollo de los países (Acemoglu y Robinson, 2012; Besley y Maitreesh, 2010). 
No es nuestro objetivo analizar la evolución de la desigualdad a nivel cuantitativo durante los últimos siglos, más bien tratamos de exponer los puntos de vista de diversos autores que sí lo han hecho, poniendo énfasis en un período de tiempo u otro, con el fin de argumentar su visión sobre este fenómeno. Una vez señalado esto, debemos reconocer que la bonanza económica que caracterizó la segunda mitad del siglo xx tuvo que ver con un desinterés por el fenómeno de la desigualdad, al menos si lo comparamos con los estudios cuantitativos que comenzaron a surgir a fines de la centuria. Desde entonces, y gracias a la evolución de los métodos estadísticos y a la recopilación de datos, el interés por reconstruir la evolución de la desigualdad durante las últimas décadas en diversos puntos del globo ha concentrado los esfuerzos de numerosos académicos. En términos generales, podemos hablar de dos visiones sobre la desigualdad en cierto modo contrapuestas. En primer lugar, los trabajos de economistas como Th. Piketty, J. Stiglitz, G. Zucman o E. Saez tienden a mostrar una visión pesimista sobre la desigualdad. Por otro lado, los planteamientos de otros economistas, como B. Milanovic, aportan un punto de vista menos crítico con el fenómeno de la desigualdad durante las últimas décadas.

Autores como van Zanden (2014) o Piketty se han preocupado por analizar la evolución de la desigualdad en Francia yen otros países occidentales durante los últimos dos siglos, teniendo en cuenta los efectos de la industrialización y de otros procesos. Los resultados de sus trabajos (Piketty, Postel-Vinay y Rosenthal, 2006) muestran que durante este período la desigualdad se ha reducido, si bien existen motivos de peso que explican esta tendencia, como pueden ser la intensidad en el crecimiento económico occidental a niveles desconocidos en la historia de la humanidad, los avances técnicos o el desarrollo de políticas económicas y de herramientas como la fiscalidad progresiva (Piketty, 2001). No obstante, la crisis económica que se instaló a nivel global hace casi una década impulsó la aparición de nuevos estudios por parte de los mismos autores, entre ellos el conocido Le Capital au XXIe siècle de Piketty, en el que se alertaba, como hicieron otros en el siglo XIX, de la potencial desigualdad que iba a generar una economía que primaba los rendimientos del capital frente a los rendimientos del trabajo. Con una orientación similar, un año antes J. Stiglitz (2012) publicó una obra de divulgación The Price of Inequality: How Today's Divided Society Endangers Our Future en la que, en base al ejemplo norteamericano, ponía en cuestión un sistema donde los más ricos estaban dispuestos a erosionar herramientas redistributivas basadas en la fiscalidad, además de imponer su poder de mercado, con el fin de perpetuar la desigualdad que tradicionalmente ha caracterizado al mundo y a las economías occidentales.

En el año 2016, el economista B. Milanovic publicó Global inequality: A New Approach for the Age of Globalization. La principal aportación se resume en un gráfico: el conocido como «Elefante de Milanovic». La curva resultante representa el incremento de la renta de todos los grupos de población, el cual ha sido notoriamente desigual. El gráfico muestra que la globalización y el progreso técnico han tenido consecuencias 
contradictorias para la desigualdad: esta se ha reducido entre los países del mundo, sin embargo, dentro de cada uno de ellos tiende a aumentar en múltiples ocasiones. Es decir, por una parte, se ha producido una convergencia externa notablemente positiva y por otra una divergencia interna que, como se ha demostrado empíricamente, resulta negativa para el desarrollo económico y social y que ha afectado en especial a las clases medias de las economías desarrolladas. A diferencia de visiones más enfocadas hacia una política fiscal redistributiva, las políticas que propone Milanovic para paliar el incremento de la desigualdad dentro de cada país van encaminadas hacia una mejora en el capital humano o la difusión del capital de manera más amplia a través de, por ejemplo, el aumento en el impuesto de sucesiones o la distribución de acciones entre los trabajadores de las empresas. En esta misma línea de escepticismo sobre los efectos de la desigualdad en nuestro tiempo, economistas como X. Sala-i-Martín (2006), afirman que, al menos durante las décadas de 1980-1990 la desigualdad a nivel mundial ha decrecido, rebajando el nivel de alerta.

Más allá de estas posturas, las visiones en torno al fenómeno de la desigualdad son muy variadas. Por ejemplo, economistas como L. Katz y C. Goldin (2010) han propuesto nuevos enfoques sobre el tema, no tan centrados en la desigualdad vía capital, defendiendo que las diferencias entre países tienen mucho que ver con el desarrollo del conocimiento. Por lo tanto, según ellos, la desigualdad en nuestros días guarda una relación directa con elementos que hasta ahora no se han tenido en cuenta, como el progreso tecnológico, sin que este sea demonizado y más bien visto como una oportunidad para reducir las diferencias en el mundo.

En definitiva, en la actualidad existe una gran diversidad de opiniones acerca de las causas y de los efectos de la desigualdad, sin llegar a un consenso claro por parte de los académicos. Mientras que los estudios sobre la propiedad privada podrían haber llegado a su estado de madurez, el análisis sobre la desigualdad desde diversos enfoques -economía, historia, sociología, antropología...- está pendiente de futuras aportaciones que permitan avanzar en el conocimiento de las causas y consecuencias de este fenómeno en nuestra sociedad y en la del futuro.

\section{CONCLUSIONES}

El principal objetivo de este trabajo era intentar establecer una relación entre propiedad privada y desigualdad a través de las reflexiones de teóricos y pensadores que, a lo largo de más de dos milenos, se preocuparon por exponer sus ideas y el resultado de sus indagaciones sobre estas dos realidades. Podríamos decir que, en términos generales, sí existe una relación, más o menos explícita según el autor y la época, entre la consolidación de la propiedad privada y el desarrollo de la desigualdad económica y social. No obstante, esta afirmación no deja de ser generalista y contiene múltiples matices. 
En lo que respecta a la propiedad privada, hemos constatado cómo el pensamiento económico occidental ha tendido hacia la defensa de la misma. Las razones que han aducido muchos pensadores desde Aristóteles hasta nuestros días eran varias. En primer lugar, las visiones más antropológicas fundamentaron su defensa de la propiedad privada en base a algunos atributos de la propia naturaleza humana, por ejemplo, en el gusto por la posesión, en el egoísmo, etc. Otros, desde Aristóteles a Smith, pasando por los representantes de la Escolástica, expusieron razones más racionales que tenían que ver con que la propiedad privada permitía un mejor cuidado de los recursos escasos a través de una administración y gestión más eficientes. Todo ello debía impulsar el desarrollo económico de los territorios gracias a la generación de riqueza y, por otra parte, permitía mantener la paz social, evitando los conflictos propios generados por el uso de la propiedad común. La consolidación de la propiedad no implicaba para estos autores la ausencia de conflictos, sin embargo, a partir del nacimiento de instituciones como la ley, la justicia y el gobierno, la paz y el bien común estaban asegurados.

Pocos pensadores han criticado la propiedad privada de forma abierta y, sobre todo, pocos han defendido la total desaparición de la misma. Precisamente, la mayor parte de estos autores son los que han puesto el acento sobre la relación causaefecto entre propiedad privada y desigualdad. Las visiones de los "semisocialistas», de los comunistas utópicos o de Marx y sus seguidores son el mejor ejemplo de lo anteriormente dicho.

Al igual que la propiedad privada, las reflexiones sobre la desigualdad han estado presentes en los escritos de los académicos y pensadores desde la Antigüedad hasta nuestros días; si bien, a diferencia de lo que apreciamos en el caso de la propiedad privada, sus análisis apenas profundizaron en la cuestión. En sus escritos, la consecución del bien común y de la paz social parecía diluir su preocupación por la desigualdad que, por otra parte, era compleja de analizar dada la escasez de datos y de metodologías aptas para su estudio.

Hoy, la propiedad privada y la desigualdad parecen seguir caminos distintos en el ámbito académico. La primera se ha consolidado como una institución cuasi sacralizada en los sistemas económicos actuales, salvo en casos excepcionales. Por otro lado, el estudio de la desigualdad ha tomado un vigor hasta ahora desconocido gracias a los nuevos métodos de análisis, a la posibilidad de contar con datos $y$, sobre todo, al interés que ha generado la cuestión tras la crisis económica que aún golpea al mundo. Este fenómeno es especialmente visible en el desarrollo de proyectos de investigación nacionales e internacionales ${ }^{5}$, en la celebración de

${ }^{5}$ Como ejemplo cabe citar los proyectos dirigidos por J. K. Galbraith «Inequality Project» - University of Texas, http://utip.lbj.utexas.edu/about.html; o por G. Alfani «Economic Inequality across Italy and Europe,1300-1800» - Università Bocconi, http://www.dondena. unibocconi.it/wps/wcm/connect/cdr/centro_dondena/home/research/einite. 
conferencias, en la edición de libros o en la publicación de monográficos en revistas académicas (Cliometrica, 2017). Además, el debate ha involucrado a instituciones internacionales (FMI, BM, etc.), a premios Nobel, a importantes economistas y pensadores y, en definitiva, a toda la sociedad, y está abierto a nuevas aportaciones que nos expliquen hasta qué punto existe desigualdad en el mundo, cuál ha sido su evolución a lo largo de la historia y qué recorrido puede tener en nuestro futuro.

\section{BIBLIOGRAFÍA}

Acemoglu, D., Johnson, S. y Robinson, J. A. (2005). Institutions as a Fundamental Cause of Long-Run Growth. En P. Aghion y S. N. Durlauf, S. N. (eds.), Handbook of Economic Growth, 1A (pp. 385-472). Elsevier.

Acemoglu, D. y Robinson, J. A. (2012). Por qué fracasan los países. Los orígenes del poder, la prosperidad y la pobreza. Barcelona: Deusto.

Alfani, G. (2015). Economic inequality in northwestern Italy: a long-term view (fourteenth to eighteenth centuries). Journal of Economic History, 75, 1058-1096.

Aquino, T. de (2001). Summa Theologica. Edición dirigida por los Regentes de Estudios de las Provincias Dominicas de España.

Aristóteles, La Política. FV Eds. (2014). Traducido por Patricio de Azcárate (1873).

Atkinson, A. B. (1983). The Economics of Inequality. Oxford: Clarendon Press.

Besley, T. y Maitreesh, M. (2010). Property Rights and Economic Development. En D. Rodrik y M. Rosenzweig (eds.), Handbook of Development Economics, Vol. 5 (pp. 4525-4595). Elsevier.

Binoche, B. (2015). La raison sans l'Histoire. Paris: PUF.

Holcombe, R. G. (2014). Advanced introduction to the Austrian School of Economics. Edwar Elgar.

Hume, D. (2001). Tratado de la naturaleza humana. Albacete: Diputación de Albacete. [Versión original A Treatise of Human Nature, 1739].

Goldin, C. y Katz, L. (2010). The race between Education and technology. Harvard University Press. 
Keynes, J. M. (2008). Teoría general de la ocupación, el interés y el dinero. México: Fondo de Cultura Económica. [Primera edición original 1934].

Légé, Ph. (2006). Socialismo y utilitarismo en la economía política de John Stuart Mill. Lecturas en Economía, 64, 169-185.

Lindert, P. H. y Williamson, J. G. (2003). Does Globalization Make the World More Unequal? En M. Bordo, A. Taylor y J. G. Williamson (eds.), Globalization in Historical Perspective (pp. 227-275). Chicago: University of Chicago Press.

Locke, J. (2014). Segundo tratado sobre el Gobierno Civil (1690. Madrid: Alianza Ed. [Versión original publicada en 1689].

Marx, K. (1932). Manuscritos Económicos y filosóficos de 1844.

Mark, K. y Engels, F. (1849). Manifiesto Comunista.

Milanovic, B., Lindert, P. y Williamson, J. G. (2011). Pre-industrial Inequality. The Economic Journal, 121(551), 255-272.

Milanovic, B. (2012). Global Income Inequality by the Numbers: in History and Now. An Overview. Policy Research Working Paper, no 6259. World Bank.

Milanovic, B. (2016). Global Inequality. A New Approach for the Age of Globalization. Harvard University Press.

Mises, L. von (1961). On Equality and Inequality. Modern Age, Spring Issue, pp. 139147.

North, D. (1990). Institutions, Institutional Change and Economic Performance. Cambridge University Press.

Perky, J. (2016). The Political Economy of Progress. John Stuart Mill and Modern Radicalism. Oxford: Oxford University Press.

Piketti, Th. (2001). Les inégalités dans le long terme. En VV. AA., Inégalités économiques (pp. 137-204). Paris: La Documentation française.

Piketty, Th. (2013). Le Capital au XXIe siècle. Seuil.

Platón, La República. 
Reis, J. (2017). Deviant behaviour? Inequality in Portugal 1565-1770. Cliometrica, 11(3), 297-319.

Roncaglia, A. (2006). La riqueza de las ideas. Una historia del pensamiento económico. Zaragoza: Prensas Universitarias de Zaragoza.

Rothbard, M. (1999). Historia del pensamiento económico. Madrid: Unión Editorial. 2 vols. [Versión original, An Austrian Perspective on the History of Economic Thought, 1995].

Rousseau, J. J. (1923). Discurso sobre el origen de la desigualdad entre los hombres. Madrid: Calpe. [Versión original, Discours sur l'origine et les fondements de I'inégalité parmi les hommes, 1754].

Sala i Martín, X. (2006). The World Distribution of Income. Falling Poverty and Convergence, Period. Quarterly Journal of Economics, 121(2), 351-397.

San Emeterio, N. (2002). La doctrina económica de la propiedad: de la Escolástica a Adam Smith. Tesis Doctoral. Universidad Complutense de Madrid.

Schumpeter, J. A. (2015). Historia del Análisis Económico. Barcelona: Ariel. [Versión original, History of Economic Analysis, 1954].

Smith, Adam (1869). Lectures on Justice, Police, Revenue and Arms, delivered in the University of Glasgow, by Adam Smith. Reported by a Student in 1763 and edited with an Introduction and notes, by Edwin Cannan. Oxford: Clarendon Press. 31/8/2017 - http://oll.libertyfund.org/titles/2621

Soto, D. de (1968). De la Justicia y del Derecho (De lustitia et lure). Madrid: Instituto de Estudios Políticos.

Stiglitz, J. (2012). The Price of Inequality: How Today's Divided Society Endangers Our Future. W. W. Norton \& Company.

Temkin, L. S. (1993). Inequality. Oxford: Oxford University Press.

van Zanden, J. L. y van Leeuwen, B. (2012). Persistent but not consistent: the growth of national income in Holland 1347 -1807. Explorations in Economic History, 49, 119-130. 
van Zanden, J. L., Baten, J., Foldvari, P. y van Leeuwen, B. (2014). The Changing Shape of Global Inequality 1820-2000; Exploring a New Dataset. Review of Income and Wealth, 60, 279-297.

Venning, C. (1976). Hume on Property, Commerce, and Empire in the Good Society: The Role of Historical Necessity. Journal of the History of Ideas, 37(1), 79-92.

Vitoria, F. de (1937). Comentarios a la Secunda Secundae de Santo Tomás, R.P. Salamanca: Vicente Beltrán de Heredia Ed., Tomo III. 